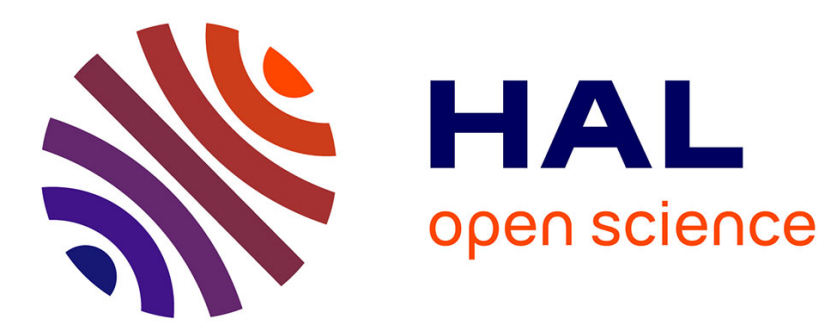

\title{
The Hoede-Bakker index modified to the Shapley-Shubik and Holler-Packel indices
}

Agnieszka Rusinowska

\section{To cite this version:}

Agnieszka Rusinowska. The Hoede-Bakker index modified to the Shapley-Shubik and Holler-Packel indices. Group Decision and Negotiation, 2010, 19 (6), pp. 543-569. halshs-00406430

\section{HAL Id: halshs-00406430 \\ https://shs.hal.science/halshs-00406430}

Submitted on 22 Jul 2009

HAL is a multi-disciplinary open access archive for the deposit and dissemination of scientific research documents, whether they are published or not. The documents may come from teaching and research institutions in France or abroad, or from public or private research centers.
L'archive ouverte pluridisciplinaire HAL, est destinée au dépôt et à la diffusion de documents scientifiques de niveau recherche, publiés ou non, émanant des établissements d'enseignement et de recherche français ou étrangers, des laboratoires publics ou privés. 


\section{The Hoede-Bakker index modified to the Shapley-Shubik and Holler-Packel indices}

\section{AGNIESZKA RUSINOWSKA}

GATE, CNRS - Université Lumière Lyon 2

93, Chemin des Mouilles - B.P.167, 69131 Ecully Cedex, France

E-mail: rusinowska@gate.cnrs.fr

Running title: Hoede-Bakker index modified to other indices 


\begin{abstract}
We present some modifications of the Hoede-Bakker index defined in a social network in which players may influence each other. Due to influences of the other actors, the final decision of a player may be different from his original inclination. The modifications presented in the paper are defined for an arbitrary probability distribution over all inclination vectors. In particular, they concern the situation in which the inclination vectors may be not equally probable. Furthermore, by assuming special probability distributions over all inclination vectors, we construct modifications of the Hoede-Bakker index that coincide with the ShapleyShubik index and with the Holler-Packel index, respectively. We present a practical example in which the concepts in question are applied to Dutch parties, and a theoretical example in which we show how the modifications can be calculated.
\end{abstract}

Key words: Hoede-Bakker index, inclination vector, probability distribution, Shapley-Shubik index, HollerPackel index

\title{
1 Introduction
}

The point of departure for this paper is the concept of decisional power (Hoede and Bakker 1982), called also the Hoede-Bakker index. This concept is defined for calculating 'power' in a social network, in which players make an acceptance-rejection decision, and they may influence each other when making such a decision. One of the main notions in this framework is the concept of an inclination vector which indicates the inclinations of all players to say 'yes' or 'no'. Due to the influence among players, the (final) decision of a player may be different from his (original) inclination. Based on the decisions of all players, a group decision ('yes' or 'no') is made. In Hoede and Bakker (1982) it is assumed that all inclination vectors are equally probable. In Rusinowska and De Swart (2007), some properties of the Hoede-Bakker index have been analyzed, with a focus on the postulates for power indices and the voting power paradoxes displayed by this index. In Rusinowska and De Swart (2006), the authors generalize the Hoede-Bakker index, by relaxing one of the originally imposed assumptions. As has been shown in Rusinowska (2008), this generalization measures a kind of 'net' Success, i.e., 'Success - Failure', but if all inclination vectors are equally probable, then this generalization happens to measure 'Decisiveness', and consequently coincides with the absolute Banzhaf index (Banzhaf 1965). By a successful player (given an inclination vector) we mean a player whose inclination coincides with the group decision. A player is said to be decisive if he is successful and changing his inclination makes the group decision change as well. Success (Decisiveness) of a player is defined as a probability that the player is successful (decisive). In Rusinowska and De Swart (2006) also some modifications of the decisional power that coincide respectively with the Rae index (Rae 1969), the Coleman indices (Coleman 1971, 1986), and the König-Bräuninger index (König and Bräuninger 1998), are proposed. These modifications are defined under the assumption that all inclination vectors are equally probable.

The aim of this paper is to introduce and analyze modified indices, based on the Hoede-Bakker set-up, that would measure decisional power in a social network in which inclination vectors do not have to be equally probable. To the best of our knowledge, such modifications in networks have not been introduced before. Moreover, since the assumption of the uniform probability distribution over all inclination vectors is quite restrictive, investigating the modified indices that allow for an arbitrary probability distribution is important, both from a theoretical point of view as well as from a point of view of the applicability of the measure in question. In the paper, first we propose four modifications of the Hoede-Bakker index for an arbitrary probability distribution over all inclination vectors. The most straightforward modification, obtained just by incorporating an arbitrary probability distribution into the definition of the Hoede-Bakker index, does not measure Decisiveness in general. Nevertheless, we deliver a necessary and sufficient condition under which this modification coincides with Decisiveness. Obviously, this condition is satisfied if all inclination vectors are equally probable. We also define three other modifications of the index, that are not that 'elegant' and straightforward as the modification mentioned above, but they are equal either to a probability that 
a player is decisive or to conditional probabilities that a player is decisive if he votes 'yes' ('no'), for an arbitrary probability distribution. Apart from defining the general modifications of the Hoede-Bakker index, by choosing 'proper' probability distributions over all inclination vectors, we also define 'à la Shapley-Shubik' and 'à la Holler-Packel' indices for a social network. To be more precise, we define three modifications of the decisional power index that coincide with the Shapley-Shubik index (Shapley and Shubik 1954), and a modification which coincides with the Holler-Packel index (Holler 1982, Holler and Packel 1983).

When measuring 'strength' of political parties, the question about the motivation behind the coalition or cabinet formation appears. In the framework of simple games, the concepts of the dominant player (Peleg 1981; see also Van Deemen 1989, 1997) and the central player (Einy 1985; Van Deemen 1991, 1997) have been developed. While the dominant player is a 'policy blind' or 'office seeking' concept, the central player is a 'policy oriented' or 'policy seeking' concept. A player $k$ is said to be a dominant player if there are disjoint coalitions $A, B$, such that $k \notin A \cup B, A \cup B$ is not a winning coalition, but $A \cup k$ and $B \cup k$ are winning coalitions. In order to find the central player of a game, the players must be ordered on a relevant policy dimension. The most simple way to get such an order of political parties is to use the 'left-right' scale (see, e.g., Morgan 1976). Player $k$ is said to be a central player if the connected coalition to the left of $k$ as well as the connected coalition to the right of $k$ can turn into a winning coalition only when $k$ joins this coalition. In Van Roozendaal (1993) the concepts of the dominant player and the central player have been applied to an analysis of Dutch parliament. In particular, the author analyzed all cabinets in the Netherlands between 1946 and 1989, and he came to the conclusion that '.. the cabinet formation game in Dutch politics seems to be much better described in terms of policy seeking motivation, as provided for in the central player theory, than by office seeking motivations as provided for in the dominant player theory' (Van Roozendaal 1993: 46-47). Nevertheless, one could easily imagine a cabinet or coalition formation whose driving force would be better described in terms of office seeking motivations. And this brings us to a wide range of possibilities of the Hoede-Bakker index concerning this issue. We like to stress that one of the advantages of the (modified) Hoede-Bakker index is related to the question of parties' motivations. Depending on how we define the influence, the Hoede-Bakker index can cover both 'policy blind' and 'policy seeking' motivations.

In the voting power literature, a conceptual debate on power indices (such as the Shapley-Shubik and Banzhaf indices) has been conducted in the course of more than ten years. The classical power indices have been criticized on the grounds that they do not take into account players' preferences (see, Garrett and Tsebelis 1996, 1999a, 1999b, 2001; Tsebelis and Garrett 1996; but also Steunenberg et al. 1999; Napel and Widgrén 2001, 2002, 2004; Hosli 1997). The authors argue that since power indices are derived from cooperative game theory, they do not take into account the strategic aspects of power in an institution, and hence they are not appropriate to analyze the distribution of power. Consequently, several developments of the classical power indices have been proposed, like introducing the so called strategic power indices derived from non-cooperative game theory (Steunenberg et al. 1999), incorporating strategic aspects or preferences into the classical power indices (Napel and Widgrén 2001, 2002; Hosli 1997, 2002), developing a unified framework which combines both cooperative and non-cooperative approaches (Napel and Widgrén 2002). In Braham and Holler (2005), this conceptual debate on power indices has been examined, and the impossibility of a preference-based power index has been discussed. The authors argue that if power is the ability of $k$ to affect an outcome, then a measure of $k$ 's power must exclude any reference to $k$ 's preference (behavioral content) with respect to affecting that outcome' ('core theorem of the measurement of power', Braham and Holler 2005: 146). Napel and Widgrén (2005) reply to these concerns on preference-based measures of power. They argue that preferences are important in determining social interactions, and therefore the preferences are also valuable in analyzing power. In this reply the authors present several convincing arguments in favor of a preference-based analysis.

Related to the debate whether a preference-based power index is conceptually meaningful, we like to stress the universal character of the Hoede-Bakker index. On the one hand, we can treat players' inclinations as preferences, and then according to Braham and Holler (2005), the (generalized or modified) Hoede-Bakker index (as based on voters' inclinations) could not be treated as a measure of power (defined as the ability of a player to affect an outcome). On the other hand, it is possible to bring the concept of the (generalized) HoedeBakker index closer to the measurement of power as defined above. In Rusinowska (2008) we propose the 
not-preference-based generalized Hoede-Bakker index, where feasible strategies instead of players' inclinations are considered. Instead of assuming that a player has the inclination to say 'yes' or 'no', we assume that the player chooses a feasible strategy (which can be called just a strategy) from among two possibilities: I will say 'yes', or I will say 'no'. Choosing a strategy by a player is equivalent to the preliminary decision of the player (i.e., his decision before any influence is executed) either to say 'yes' or to say 'no'. As soon as a player has made a preliminary decision about which strategy he chooses, a confrontation with real life takes place, which may bring a lot of influences of the others. Due to such influences, the final decision of a player may be different from his feasible strategy (meant as his preliminary decision). In Rusinowska (2008) we argue that the inclinations of a player do not have to coincide with strategies of the player.

The paper is organized as follows. Section 2 concerns the probabilistic approach to power indices. In Section 3, the generalized Hoede-Bakker index is recapitulated. In Section 4 we propose several modifications of the generalized Hoede-Bakker index for an arbitrary probability distribution over the inclination vectors. In the two following sections we choose the probability distributions under which the modifications defined become an 'à la Shapley-Shubik index' and an 'à la Holler-Packel index' for a social network. While in Section 5 we present modifications of the generalized Hoede-Bakker index that coincide with the ShapleyShubik index, Section 6 concerns a modification coinciding with the Holler-Packel index. In Section 7 we present two examples that illustrate the concepts introduced. In Section 7.1 a practical example in which the concepts in question are applied to Dutch parties is delivered. Section 7.2 presents a theoretical example, showing how to calculate the introduced modifications. In Section 8, we conclude. The paper has also an Appendix containing the proofs of all propositions.

\section{Probabilistic approach to power indices}

There are basically two approaches to power indices: the axiomatic approach and the probabilistic one. In this research, we apply the probabilistic model for measuring 'Decisiveness' in voting situations presented in Laruelle and Valenciano (2005). Below, we present the main concepts of this model.

Let $N=\{1,2, \ldots, n\}$ be the set of voters who have to vote ('yes' with abstention included or 'no') on a submitted proposal. A vote configuration $S$ is the result of voting in which all voters in $S$ vote 'yes', and all voters in $N \backslash S$ vote 'no'. Hence, $k \in S$ means that voter $k$ votes 'yes'. A winning configuration is a vote configuration leading to the passage of the proposal in question. Let $W$ be the set of winning configurations. The pair $(N, W)$ is called an $N$-voting rule. The following conditions are imposed: (i) $N \in W$; (ii) $\emptyset \notin W$; (iii) If $S \in W$, then $T \in W$ for any $T$ containing $S$; (iv) If $S \in W$, then $N \backslash S \notin W$. $^{1}$

A probability distribution over all possible vote configurations is assumed. It is represented by a map $p: 2^{N} \rightarrow[0,1]$, associating with each vote configuration $S \subseteq N$ its probability $0 \leq p(S) \leq 1$ to occur, where $\sum_{S \subset N} p(S)=1$. That is, $p(S)$ is the probability that all voters in $S$ vote 'yes', and all voters in $N \backslash S$ vote 'no'. Let $(W, p)$ be an $N$-voting situation, where $W$ is the voting rule to be used and $p$ is the probability distribution over vote configurations, and let $k \in N$. Then:

$$
\begin{gathered}
\Phi_{k}(W, p):=\operatorname{Prob}(k \text { is decisive })=\sum_{\substack{S: k \in S \in W \\
S \backslash\{k\} \notin W}} p(S)+\sum_{\substack{S: k \notin S \notin W \\
S \cup\{k\} \in W}} p(S)= \\
\sum_{\substack{S: k \in S \in W \\
S \backslash\{k\} \notin W}}(p(S)+p(S \backslash\{k\})) \\
\Phi_{k}^{+}(W, p):=\operatorname{Prob}\left(k \text { is decisive } \mid k \text { votes 'yes' }{ }^{\prime}\right)=\frac{\sum_{\substack{S: k \in S \in W \\
S \backslash\{k\} \notin W}} p(S)}{\sum_{S: k \in S} p(S)} \\
\Phi_{k}^{-}(W, p):=\operatorname{Prob}\left(k \text { is decisive } \mid k \text { votes 'no' }{ }^{\prime}\right)=\frac{\sum_{\substack{S: k \notin S \notin W \\
S \cup\{k\} \in W}} p(S)}{\sum_{S: k \notin S} p(S)} .
\end{gathered}
$$

\footnotetext{
${ }^{1}$ This condition is not necessary in this model.
} 


\section{The generalized decisional power index}

The concept of decisional power or the Hoede-Bakker index (Hoede and Bakker 1982) has been generalized in Rusinowska and De Swart (2006). In this section, we recapitulate the definition of the generalized HoedeBakker index. We consider a social network with $n \geq 2$ players (actors, voters) who are to make a 'yes'-'no' decision, and they may influence each other when making their decisions. Let $N=\{1, \ldots, n\}$ be the set of all players. Each actor has an inclination either to say 'yes' (denoted by +1 ) or 'no' (denoted by -1 ). Let $i$ be an inclination vector (i.e., an $n$-vector consisting of ones and minus ones and indicating the inclinations of the actors), and let $I$ denote the set of all inclination vectors. Of course, $|I|=2^{n}$. Due to influence of the others, the final decision of an actor may be different from his original inclination. Each inclination vector $i \in I$ is then transformed into a decision vector $b$ which is also an $n$-vector consisting of ones and minus ones and indicating the decisions made by all players. Formally, there is an operator $B: I \rightarrow B(I)$, that is, $b=B i$, where $B(I)$ denotes the set of all decision vectors. We also introduce the group decision $g d: B(I) \rightarrow\{+1,-1\}$ which is a function defined on the decision vectors $b$, having the value +1 if the group decision is 'yes', and the value -1 if the group decision is 'no'. We introduce the following notation. Let

$$
\begin{gathered}
i \leq i^{\prime} \Longleftrightarrow\left\{k \in N \mid i_{k}=+1\right\} \subseteq\left\{k \in N \mid i_{k}^{\prime}=+1\right\} \\
i<i^{\prime} \Longleftrightarrow\left[i \leq i^{\prime} \wedge i \neq i^{\prime}\right] .
\end{gathered}
$$

Moreover, let $i^{\emptyset}=(-1, \ldots,-1)$ denote the inclination vector with negative inclinations of all voters, and let $i^{N}=(+1, \ldots,+1)$ denote the inclination vector with positive inclinations of all voters. Let $g d(B)$ be the composition of $B$ and $g d$. In Rusinowska and De Swart (2006), the following three conditions have been imposed:

$$
\begin{gathered}
\forall i \in I \forall i^{\prime} \in I\left[i \leq i^{\prime} \Rightarrow g d(B i) \leq g d\left(B i^{\prime}\right)\right] \\
g d\left(B i^{N}\right)=+1, \quad g d\left(B i^{\emptyset}\right)=-1,
\end{gathered}
$$

and the following definition has been introduced. Given $g d(B)$, the generalized Hoede-Bakker index (the generalized decisional power index) of player $k \in N$ is given by

$$
G H B_{k}(g d(B)):=\frac{1}{2^{n}} \cdot\left(\sum_{\left\{i: i_{k}=+1\right\}} g d(B i)-\sum_{\left\{i: i_{k}=-1\right\}} g d(B i)\right) .
$$

As mentioned before, the generalized Hoede-Bakker index is a measure of Success - Failure (Rusinowska 2008), and this index measures Decisiveness (that is, it coincides with the absolute Banzhaf index) if all inclination vectors are equally probable (Rusinowska and De Swart 2006).

\section{Modifications of the Hoede-Bakker index for an arbitrary prob- ability distribution}

In modifications of the Hoede-Bakker index proposed in Rusinowska and De Swart (2006), all inclination vectors are assumed to be equally probable, that is, the probability distribution over all inclination vectors is the following:

$$
p^{*}(i):=\frac{1}{2^{n}} \text { for all } i \in I .
$$

In this paper, we relax assumption (9) and propose several modifications of the generalized Hoede-Bakker index that are defined for an arbitrary probability distribution $p: I \rightarrow[0,1]$ over all inclination vectors.

Let $k \in N$ and $i=\left(i_{1}, \ldots, i_{n}\right)$. By $i^{k}=\left(i_{1}^{k}, \ldots, i_{n}^{k}\right)$ we denote the inclination vector resulting from $i$ by changing only $i_{k}$, i.e.,

$$
i_{j}^{k}=\left\{\begin{array}{ccc}
i_{j} & \text { if } & j \neq k \\
-i_{j} & \text { if } & j=k
\end{array} .\right.
$$


Similar as in Rusinowska and De Swart (2006), we introduce a bijection $f: I \rightarrow 2^{N}$ between inclination vectors and vote configurations (coalitions) which assigns to each inclination vector $i$ the coalition consisting of all voters with positive inclination in $i$, i.e., for all $i \in I$,

$$
f(i)=\left\{k \in N \mid i_{k}=+1\right\} .
$$

Let for each $i \in I,|i|$ denote the number of all voters with positive inclination in $i$,

$$
|i|:=|f(i)|=\left|\left\{k \in N \mid i_{k}=+1\right\}\right| .
$$

Moreover, given $g d(B)$, we say that:

- coalition $f(i)$ (equivalently, inclination vector $i \in I$ ) is winning $(f(i) \in W)$ iff $g d(B i)=+1$;

- $f(i)$ (equivalently, $i \in I$ ) is losing iff $g d(B i)=-1$;

- $f(i)$ (equivalently, $i \in I$ ) is minimal winning iff $g d(B i)=+1$ and for each $i^{\prime}<i, g d\left(B i^{\prime}\right)=-1$.

In other words, an inclination vector $i$ is winning if it leads to a positive group decision, and it is losing otherwise (i.e., if it leads to a negative group decision). A minimal winning inclination vector is a winning inclination vector such that if at least one player with positive inclination changes his inclination to the negative one, then the group decision changes to 'no'. By $I^{m w}$ we denote the set of all minimal winning inclination vectors, that is,

$$
I^{m w}:=\left\{i \in I \mid g d(B i)=+1 \wedge \forall i^{\prime}<i\left[g d\left(B i^{\prime}\right)=-1\right]\right\} .
$$

$\left|I^{m w}\right|$ denotes the number of minimal winning inclination vectors. We assume that the probability distribution $p$ over all inclination vectors is the same as the probability distribution over all corresponding coalitions, that is,

$$
\forall i \in I[p(i)=p(f(i))]
$$

and, consequently,

$$
\sum_{i \in I} p(i)=1
$$

where $0 \leq p(i) \leq 1$ denotes the probability that the inclination vector $i$ occurs, and $f$ is defined in (11). We impose conditions (6) and (7), and by replacing equal probabilities of the inclination vectors in the definition of the generalized Hoede-Bakker index by an arbitrary probability distribution, we introduce the following straightforward modification of this index.

Definition 4.1 Given $g d(B)$ and probability distribution $p$ over all inclination vectors, for each $k \in N$ :

$$
\Gamma_{k}(g d(B), p):=\sum_{\left\{i: i_{k}=+1\right\}} p(i) \cdot g d(B i)-\sum_{\left\{i: i_{k}=-1\right\}} p(i) \cdot g d(B i) .
$$

Since $g d(B i) \in\{-1,+1\}$ for $i \in I$, we get, for each $k \in N, \Gamma_{k}(g d(B), p) \leq 1$. Unfortunately, for an arbitrary $p, \Gamma_{k}(g d(B), p)$ is not a power index measuring Decisiveness of player $k$ any more, where (as mentioned before) Decisiveness of $k$ means that by changing the inclination of $k$, the group decision changes as well. In particular, $\Gamma_{k}(g d(B), p)$ may be even negative. Of course, by assuming some (sometimes artificial) conditions, we get $\Gamma_{k}(g d(B), p) \geq 0$ for $k \in N$. One may, for instance, impose a kind of 'generalized' version of $(6)$, that is,

$$
\forall i \in I \forall i^{\prime} \in I\left[i \leq i^{\prime} \Rightarrow p(i) \cdot g d(B i) \leq p\left(i^{\prime}\right) \cdot g d\left(B i^{\prime}\right)\right]
$$

and then get $\Gamma_{k}(g d(B), p) \geq 0$ for each $k \in N$. However, not for all compositions $g d(B)$ and probability distributions $p$, condition (17) is satisfied. Let us take two inclination vectors $i, i^{\prime}$ with positive probabilities to occur such that $i \leq i^{\prime}$, i.e., $i^{\prime}$ results from $i$ by enlarging (or not changing) the set of potential 'yes' voters. 
First of all, according to (17), if $i$ is winning, then $i^{\prime}$ must be winning as well. Moreover, if both $i$ and $i^{\prime}$ are winning, then $p(i) \leq p\left(i^{\prime}\right)$, i.e., the inclination vector $i^{\prime}$ is at least as probable as $i$. On the other hand, if both $i$ and $i^{\prime}$ are losing, then $p(i) \geq p\left(i^{\prime}\right)$, i.e., $i$ is at least as probable as the inclination vector $i^{\prime}$. This seems to suggest that the relation between probabilities of voters' inclinations depends on the final results of voting, that is, on the group decisions these inclinations lead to. In other words, the more potential 'yes' voters form a winning coalition, the more probable this coalition is, and the more potential 'yes' voters form a losing coalition, the less probable such a coalition is.

The question arises when $\Gamma_{k}(g d(B), p)$ is equal to the probability that voter $k$ is decisive. We assume that a coalition is winning if and only if the inclination vector in which all members of that coalition have positive inclination and all members outside the coalition have negative inclination, leads to the positive group decision, i.e.,

$$
S=f(i) \in W \Leftrightarrow g d(B i)=+1 .
$$

Given the relation (18) between $W$ and $g d(B)$, we have

Proposition 4.1 Given $g d(B), W$ and $p$, for each $k \in N$

$$
\Gamma_{k}(g d(B), p)=\Phi_{k}(W, p) \Leftrightarrow \sum_{\left\{i: i_{k}=+1\right\}}\left(p(i)-p\left(i^{k}\right)\right) \cdot\left(g d(B i)+g d\left(B i^{k}\right)\right)=0 .
$$

According to condition (19), if, in particular, all inclination vectors are equally probable, that is, the probability distribution satisfies condition $(9)$, then $\Gamma_{k}(g d(B), p)$ does measure Decisiveness of voter $k$. This is, of course, consistent with the result proved in Rusinowska and De Swart (2006) that the generalized Hoede-Bakker index $G H B_{k}(g d(B))$ recapitulated in (8) is equal to the probability that player $k$ is decisive.

Since $\Gamma_{k}$ coincides with Decisiveness of $k$ only under condition (19), we propose also other modifications of the Hoede-Bakker index. In these modifications, for each player $k$, we take into account at the same time the group decisions resulting from some inclination vectors $i$, and the group decisions resulting from $i^{k}$ (that is, from the inclination vectors obtained from $i$ by changing only the inclination $i_{k}$ of player $k$ ). This is related to the definition of a decisive player who, by changing solely his inclination, makes the group decision change as well. As before, we impose conditions (6) and (7), and introduce the following definitions.

Definition 4.2 Given $g d(B)$ and probability distribution $p$ over all inclination vectors, for each $k \in N$ :

$$
\begin{gathered}
\Psi_{k}(g d(B), p):=\sum_{\left\{i: i_{k}=+1\right\}} \frac{p(i)+p\left(i^{k}\right)}{2} \cdot\left(g d(B i)-g d\left(B i^{k}\right)\right) \\
\Psi_{k}^{+}(g d(B), p):=\frac{\sum_{\left\{i: i_{k}=+1\right\}} p(i) \cdot\left(g d(B i)-g d\left(B i^{k}\right)\right)}{2 \sum_{\left\{i: i_{k}=+1\right\}} p(i)} \\
\Psi_{k}^{-}(g d(B), p):=\frac{\sum_{\left\{i: i_{k}=-1\right\}} p(i) \cdot\left(g d\left(B i^{k}\right)-g d(B i)\right)}{2 \sum_{\left\{i: i_{k}=-1\right\}} p(i)} .
\end{gathered}
$$

Of course, since $g d(B i) \in\{-1,+1\}$ for $i \in I$, and by virtue of $(6)$, we have for each $g d(B), p$, and $k \in N$, $0 \leq \Psi_{k}(g d(B), p) \leq 1,0 \leq \Psi_{k}^{+}(g d(B), p) \leq 1$, and $0 \leq \Psi_{k}^{-}(g d(B), p) \leq 1$. Although these modifications are not that 'elegant' and straightforward as $\Gamma$, they always coincide with Decisiveness of player $k$. Given (18), we have

Proposition 4.2 Given $g d(B), W$ and $p$, for each $k \in N$

$$
\begin{gathered}
\Psi_{k}(g d(B), p)=\Phi_{k}(W, p) \\
\Psi_{k}^{+}(g d(B), p)=\Phi_{k}^{+}(W, p) \\
\Psi_{k}^{-}(g d(B), p)=\Phi_{k}^{-}(W, p) .
\end{gathered}
$$


According to (23), the modification $\Psi_{k}$ is always equal to the probability that player $k$ is decisive. Result (24) says that $\Psi_{k}^{+}$is equal to the conditional probability that player $k$ is decisive under the condition that $k$ votes 'yes'. Similarly, (25) means that $\Psi_{k}^{-}$is the conditional probability that player $k$ is decisive if $k$ votes 'no'.

\section{Modifications leading to the Shapley-Shubik index}

One of the most well-known power indices presented in the literature on voting power is the Shapley-Shubik index (Shapley and Shubik 1954). Using notations from Section 2, this index is defined as follows. For a given voting rule $W$, the Shapley-Shubik index, for each $k \in N$, is given by

$$
S h_{k}(W)=\sum_{\substack{S: k \in S \in W \\ S \backslash\{k\} \notin W}} \frac{(n-|S|) ! \cdot(|S|-1) !}{n !}
$$

where $|S|$ denotes the number of voters voting 'yes'.

We would like to extend our work done in Rusinowska and De Swart (2006), in which modifications of the generalized Hoede-Bakker index that coincide with the Rae index, the Coleman indices, and the KönigBräuninger index are presented. The question appears whether by choosing 'proper' probability distributions over all inclination vectors, our modifications of the generalized Hoede-Bakker index may coincide with the Shapley-Shubik index. First, we introduce some notation. Let for $x \in N$

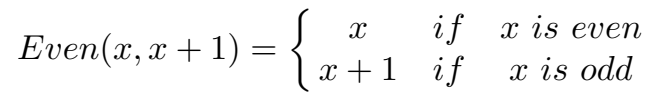

and

$$
\operatorname{Odd}(x, x+1)=\left\{\begin{array}{ccc}
x & \text { if } & x \text { is odd } \\
x+1 & \text { if } & x \text { is even }
\end{array}\right.
$$

Given (18), we have

Proposition 5.1 $\Psi_{k}\left(g d(B), p^{S h}\right)=S h_{k}(W)$ for all $k \in N, g d(B)$ and $W$ if and only if

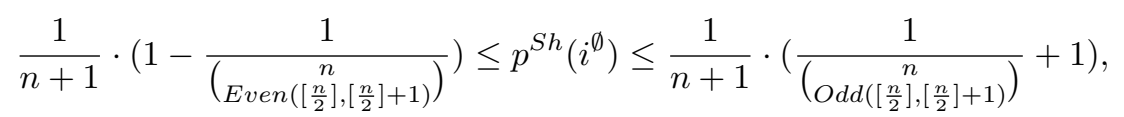

and

$$
p^{S h}(i)=\left\{\begin{array}{lll}
\frac{1}{n+1} \cdot\left(\frac{1}{\left(\begin{array}{c}
n \\
|i|
\end{array}\right)}-1\right)+p^{S h}\left(i^{\emptyset}\right) & \text { if } & |i| \text { is even } \\
\frac{1}{n+1} \cdot\left(\frac{1}{\left(\begin{array}{c}
n \\
|i|
\end{array}\right)}+1\right)-p^{S h}\left(i^{\emptyset}\right) & \text { if } & |i| \text { is odd }
\end{array}\right.
$$

In particular, $\Psi_{k}\left(g d(B), p^{S h}\right)=S h_{k}(W)$ for all $k \in N, g d(B)$ and $W$ if for each $i \in I$

$$
p^{S h}(i)=\frac{1}{n+1} \cdot \frac{1}{\left(\begin{array}{c}
n \\
|i|
\end{array}\right)} .
$$

In Proposition 5.1, we present sufficient and necessary conditions for the probability distribution under which the modification $\Psi_{k}$ of the Hoede-Bakker index coincides with the Shapley-Shubik index $S h_{k}$ of player $k$. According to these conditions, the probability of the inclination vector $i$ takes one of the two forms, depending on whether the number of potential 'yes' voters in $i$ is even or odd; see equation (30). Each possibility for $p^{S h}(i)$ given in (30) depends on the probability of the inclination vector $i^{\emptyset}$ in which all players have negative inclination. Inequality (29) gives the range for the probability of $i^{\emptyset}$. In particular, $\Psi_{k}$ and $S h_{k}$ coincide when the probability $p^{S h}\left(i^{\emptyset}\right)$ is equal to $\frac{1}{n+1}$ (which of course belongs to the interval given in $(29)$ ). In such a case, the probability distribution $p^{S h}(i)$ over all inclination vectors $i$ is given in (31). 
Proposition $5.2 \Psi_{k}^{+}\left(g d(B), p^{S h}\right)=S h_{k}(W)$ for all $k \in N, g d(B)$ and $W$ iff $0 \leq p^{S h}\left(i^{\emptyset}\right)<1$ and for any $i \neq i^{\emptyset}$

$$
p^{S h}(i)=\left(1-p^{S h}\left(i^{\emptyset}\right)\right) \cdot \frac{\frac{1}{|i|}}{\sum_{t=1}^{n} \frac{1}{t}} \cdot \frac{1}{\left(\begin{array}{c}
n \\
|i|
\end{array}\right)} .
$$

In particular, $\Psi_{k}^{+}\left(g d(B), p^{S h}\right)=S h_{k}(W)$ for $k \in N, g d(B)$ and $W$ if $p^{S h}\left(i^{\emptyset}\right)=0$ and for any $i \neq i^{\emptyset}$

$$
p^{S h}(i)=\frac{\frac{1}{|i|}}{\sum_{t=1}^{n} \frac{1}{t}} \cdot \frac{1}{\left(\begin{array}{c}
n \\
|i|
\end{array}\right)} .
$$

Proposition 5.2 is similar to Proposition 5.1, but now we deliver sufficient and necessary conditions for the probability distribution under which the modification $\Psi_{k}^{+}$(defined in (21)) coincides with the Shapley-Shubik index $S h_{k}$ of player $k$. According to these conditions, the probability of the inclination vector $i^{\emptyset}$ is arbitrary (but smaller then 1), and the probability of the inclination vector $i$ also depends on the probability of $i^{\emptyset}$, and it is given in (32). In particular, $\Psi_{k}^{+}$and $S h_{k}$ coincide when the probability $p^{S h}\left(i^{\emptyset}\right)$ that all players have negative inclination is equal to 0 . In this case, (33) presents the probability distribution $p(i)$ over all inclination vectors $i$.

Proposition $5.3 \Psi_{k}^{-}\left(g d(B), p^{S h}\right)=S h_{k}(W)$ for all $k \in N, \operatorname{gd}(B)$ and $W$ iff $0 \leq p^{S h}\left(i^{N}\right)<1$ and for any $i \neq i^{N}$

$$
p^{S h}(i)=\left(1-p^{S h}\left(i^{N}\right)\right) \cdot \frac{\frac{1}{n-|i|}}{\sum_{t=1}^{n} \frac{1}{t}} \cdot \frac{1}{\left(\begin{array}{c}
n \\
|i|
\end{array}\right)} .
$$

In particular, $\Psi_{k}^{-}\left(g d(B), p^{S h}\right)=S h_{k}(W)$ for $k \in N, g d(B)$ and $W$ if $p^{S h}\left(i^{N}\right)=0$ and for any $i \neq i^{N}$

$$
p^{S h}(i)=\frac{\frac{1}{n-|i|}}{\sum_{t=1}^{n} \frac{1}{t}} \cdot \frac{1}{\left(\begin{array}{c}
n \\
|i|
\end{array}\right)} .
$$

The results presented in Proposition 5.3 are symmetric to the ones shown in Proposition 5.2, and they give sufficient and necessary conditions for the probability distribution under which the modification $\Psi_{k}^{-}$ (defined in (22)) coincides with the Shapley-Shubik index $S h_{k}$. The probability of the inclination vector $i^{N}$ under which all players have positive inclination is arbitrary (but smaller then 1). The probability of the inclination vector $i$ depends on the probability of $i^{N}$, and it is now given in (34). In particular, $\Psi_{k}^{-}$and $S h_{k}$ coincide when the probability $p^{S h}\left(i^{N}\right)$ that all players have positive inclination is equal to 0 . The probability distribution $p^{S h}(i)$ over all inclination vectors $i$ is then presented in (35).

\section{Modification leading to the Holler-Packel index}

Another power index analyzed in the literature is the Holler-Packel index (Holler 1982, Holler and Packel 1983), an index referring to minimal winning configurations. A winning configuration is minimal if it does not contain properly any other winning configuration. The Holler-Packel index is based on the following assumptions: only minimal winning configurations will be formed, all minimal winning configurations are equally probable, all voters in a minimal winning coalition get the undivided coalition value.

Let $M(W)$ be the set of all minimal winning configurations. Let $m(W)$ and $m_{k}(W)$ denote the number of minimal winning configurations and the number of minimal winning configurations containing voter $k \in N$, respectively. For a given voting rule $W$, the non-normalized Holler-Packel index, for each $k \in N$, is defined by

$$
H P_{k}(W)=\frac{m_{k}(W)}{m(W)},
$$

and the Holler-Packel index, for each $k \in N$, is given by

$$
\widetilde{H P}_{k}(W)=\frac{H P_{k}(W)}{\sum_{j \in N} H P_{j}(W)}=\frac{m_{k}(W)}{\sum_{j \in N} m_{j}(W)} .
$$


One of our modifications of the generalized decisional power index coincides with the non-normalized Holler-Packel index. We have

Proposition 6.1 $\Psi_{k}\left(g d(B), p^{H P}\right)=H P_{k}(W)$ for all $k \in N, g d(B)$ and $W$, where for each $i \in I$

$$
p^{H P}(i)=\left\{\begin{array}{cll}
\frac{1}{\left|I^{m w}\right|} & \text { if } & i \in I^{m w} \\
0 & \text { if } & i \notin I^{m w}
\end{array},\right.
$$

and $I^{m w}$ denotes the set of all minimal winning inclination vectors (as defined in (13)). According to Proposition 6.1, the modification $\Psi_{k}$ (defined in (20)) of the Hoede-Bakker index coincides with the nonnormalized Holler-Packel index $H P_{k}$ if the probability distribution over all inclination vectors is given by (38). According to condition (38), $p^{H P}$ is the uniform probability distribution among all minimal winning inclination vectors (i.e., the inclination vectors $i$ that lead to a positive group decision, but if at least one player in $i$ with positive inclination switches to a negative one, then the new inclination vector leads to a negative group decision). Consequently, equation (38) assigns probability 0 to all inclination vectors that are not minimal winning.

\section{Examples}

In order to illustrate the concept of the (modified) Hoede-Bakker index, we present two examples. In Section 7.1 an application of the Hoede-Bakker index to the Dutch data used in Van Roozendaal (1993) is shown, while in Section 7.2 we present a theoretical example showing how all the calculations related to the modified Hoede-Bakker index are made.

\subsection{Practical example}

Let us consider an example based on the structure of the Second Chamber (Tweede Kamer) of the Dutch Parliament after the elections in 1956. There were 7 parties in the parliament at that time:

PvdA - Labor Party

KVP - Catholic People's Party

ARP - Anti-Revolutionary Party

CHU - Christian Historical Union

VVD - People's Party for Freedom and Democracy

CPN - Communist Party

SGP - Political Reformed Party

and therefore,

$$
N=\{\mathrm{PvdA}, \mathrm{KVP}, \mathrm{ARP}, \mathrm{CHU}, \mathrm{VVD}, \mathrm{CPN}, \mathrm{SGP}\} .
$$

Based on a left-right scale for the postwar period in the Netherlands, developed in Morgan (1976) (see also Van Roozendaal (1993)), the following placement of the parties is constructed:

$$
\text { CPN - PvdA - KVP - ARP - CHU - VVD - SGP }
$$

Table 1 shows the Dutch parties in the Parliament $(k \in N)$ after the Dutch election in 1956, placed in the order mentioned above, together with the numbers of seats $\left(w_{k}\right)$. In 1956 the total number of seats raised from 100 to 150 .

After the 1956 election, in the beginning the cabinet consisted of PvdA (dominant party), KVP (central party), ARP, and CHU, but this cabinet fell after slightly more than 2 months. A new cabinet was constructed which consisted of KVP, ARP, and CHU only. Let us apply the concepts introduced in the paper to this Dutch parliament. 
Table 1: The Second Chamber of the Dutch Parliament (election year 1956)

\begin{tabular}{c||c|c|c|c|c|c|c}
$k \in N$ & CPN & PvdA & KVP & ARP & CHU & VVD & SGP \\
\hline$w_{k}$ & 7 & 50 & 49 & 15 & 13 & 13 & 3
\end{tabular}

Table 2: The generalized Hoede-Bakker index $G H B_{k}$ and its modification $\Gamma_{k}, k \in N$

\begin{tabular}{c||c|c|c|c|c|c|c}
$k \in N$ & CPN & PvdA & KVP & ARP & CHU & VVD & SGP \\
\hline \hline$G H B_{k}(g d(B))$ & 0 & 0.5 & 0.5 & 0.333 & 0.333 & 0.333 & 0 \\
\hline$\Gamma_{k}(g d(B), \widetilde{p})$ & 0.2 & 0.6 & 1 & 1 & 1 & 0.6 & 0.2 \\
\hline$\Gamma_{k}^{\prime}(g d(B), \widehat{p})$ & 0.333 & 0.666 & 1 & 0.833 & 0.666 & 0.333 & 0
\end{tabular}

When applying the standard power indices, we receive the information, in particular, about power, i.e., about Decisiveness of each party. Some of the modifications of the Hoede-Bakker index coincide with the standard power indices, assuming that relation (18) holds. In particular, if we additionally assume that all inclination vectors are equally probable, then the modification $\Psi_{k}$ expresses Decisiveness of player $k$.

When applying the modifications of the Hoede-Bakker index to the framework of a social network, an arbitrary probability distribution can be considered as well, and with some probability distributions, these modifications lead to the standard power indices. In particular, if (18) is satisfied and the probability of each inclination vector $i$ is given in (31), where $n=7$, then $\Psi_{k}$ coincides with the Shapley-Shubik index, while with the probability of each inclination vector $i$ given in (38), where $I^{m w}$ is the set of all minimal winning inclination vectors, $\left|I^{m w}\right|=8$, we receive the non-normalized Holler-Packel index.

Depending on how the influence function is defined, the modified Hoede-Bakker index can cover different motivations of players. Let us illustrate this point in our example. First, we assume that every party has office seeking motivations, and the decision of a party is always equal to the inclination of a coalition having in total more than 75 seats in parliament. Consequently, the decision of each party coincides with the group decision, i.e., for each $i \in I$ and $k \in N$,

$$
(B i)_{k}=\left\{\begin{array}{ccc}
+1 & \text { if } & \sum_{j: i_{j}=+1} w_{j} \geq 76 \\
-1 & \text { otherwise }
\end{array}=\operatorname{gd}(B i) .\right.
$$

Suppose that all inclination vectors are equally probable. The generalized Hoede-Bakker indices for this case are presented in Table 2. If by a successful player under $i$ we mean a player $k$ whose inclination $i_{k}$ coincides with the group decision $g d(B i)$, and by a player who fails we mean a player who is not successful, then as we already know $G H B_{k}$ measures a kind of 'net' Success of $k$ (i.e., Success - Failure). We can then conclude from Table 2 that both CPN and SGP (whose generalized Hoede-Bakker index is equal to 0) are successful in $50 \%$ of the inclination vectors, and they fail in the remaining $50 \%$. Concerning ARP, CHU, and VVD, each of these parties achieves Success in $\frac{2}{3}$ of the inclination vectors, and fails in $\frac{1}{3}$ of them. The most successful players are PvdA and KVP: their inclinations coincide with the group decision in $75 \%$ of the inclination vectors, and they are different from the group decision in $25 \%$.

Suppose now that the parties have some policy seeking motivations, and that the inclination vectors are not all equally probable. It is reasonable to assume that:

(i) The three main religious parties, KVP, ARP, and $\mathrm{CHU}^{2}$ have the same inclinations.

(ii) The inclinations of the parties are 'coherent' in the sense that considering the left-right placement of the parties, if one party, say party $k$, has an inclination different from the inclination of its 'neighbor' to the left, then all the parties to the right of $k$ have the same inclination as $k$.

\footnotetext{
${ }^{2}$ In 1977 these parties joined and formed CDA - Christian Democratic Appeal
} 
Table 3: The inclination vectors satisfying (i)-(iii), $\widetilde{I}$

\begin{tabular}{c||c}
$\left(i_{\mathrm{CPN}}, i_{\mathrm{PvdA}}, i_{\mathrm{KVP}}, i_{\mathrm{ARP}}, i_{\mathrm{CHU}}, i_{\mathrm{VVD}}, i_{\mathrm{SGP}}\right)$ & $\left(i_{\mathrm{CPN}}, i_{\mathrm{PvdA}}, i_{\mathrm{KVP}}, i_{\mathrm{ARP}}, i_{\mathrm{CHU}}, i_{\mathrm{VVD}}, i_{\mathrm{SGP}}\right)$ \\
\hline \hline$(+1,+1,+1,+1,+1,+1,+1)$ & $(-1,-1,-1,-1,-1,-1,-1)$ \\
\hline$(+1,+1,+1,+1,+1,+1,-1)$ & $(-1,-1,-1,-1,-1,-1,+1)$ \\
\hline$(-1,+1,+1,+1,+1,+1,+1)$ & $(+1,-1,-1,-1,-1,-1,-1)$ \\
\hline$(+1,+1,+1,+1,+1,-1,-1)$ & $(-1,-1,-1,-1,-1,+1,+1)$ \\
\hline$(-1,-1,+1,+1,+1,+1,+1)$ & $(+1,+1,-1,-1,-1,-1,-1)$
\end{tabular}

(iii) All inclination vectors that satisfy (i) and (ii) have the same positive probability to occur, and the probability of each of the remaining inclination vectors is equal to 0 .

Table 3 presents the set $\widetilde{I}$ of all inclination vectors with a positive probability occurring in this situation.

The probability distribution for this case is equal to

$$
\widetilde{p}(i)=\left\{\begin{array}{ccc}
0.1 & \text { if } & i \in \widetilde{I} \\
0 & & \text { otherwise }
\end{array} .\right.
$$

We can apply now the modification $\Gamma_{k}$ for the situation in which the probability distribution over all inclination vectors is given by (39). The results are presented in Table 2. As we can see from this table, by considering only 'coherent' inclinations, all players gained, i.e., the modification $\Gamma_{k}$ is greater than the generalized Hoede-Bakker index $G H B_{k}$, for each $k \in N$. In particular, thanks to the assumption (i), the three religious parties got the highest value of $\Gamma_{k}$ : their inclination always coincides with the group decision. It is also related to the position of KVP, which was the central party in this parliament. Since the connected coalition to the left of KVP as well as the connected coalition to the right of KVP can turn into a winning coalition only when KVP joins this coalition, then together with the assumptions imposed in this case, KVP, and consequently also ARP and CHU, are always successful.

We can keep the assumptions (ii) and (iii), but relax assumption (i), and instead of (i) assume that the inclination vectors in which not all three parties KVP, ARP, and CHU have the same inclinations are less probable than the other inclination vectors. Let

$$
\begin{gathered}
\widehat{I}=\{(-1,-1,-1,+1,+1,+1,+1),(+1,+1,+1,+1,-1,-1,-1), \\
(-1,-1,-1,-1,+1,+1,+1),(+1,+1,+1,-1,-1,-1,-1)\}
\end{gathered}
$$

Suppose that the set of all inclination vectors with a positive probability occurring in this situation is equal to $\widetilde{I} \cup \widehat{I}$, and the probability distribution is the following:

$$
\widehat{p}(i)=\left\{\begin{array}{rlrl}
\frac{1}{12} & \text { if } & i & \in \widetilde{I} \\
\frac{1}{24} & \text { if } & i \in \widehat{I} \\
0 & & \text { otherwise }
\end{array} .\right.
$$

We can calculate the modification $\Gamma_{k}^{\prime}$ for this case; see Table 2. KVP did not lose anything in this case, i.e., this party is always successful $\left(\Gamma_{K V P}^{\prime}(\operatorname{gd}(B), \widehat{p})=1\right)$. Moreover, both parties to the left of KVP, that is, CPN and PvdA gained in terms of 'net' Success. This can be explained by the fact that all inclination vectors in $\widehat{I}$ that are added to the considerations, count for Success of CPN and PvdA. The situation of each party to the right of KVP, that is, ARP, CHU, VVD and SGP became worse now: they all lose in terms of 'net' Success, but ARP lost relatively less than the remaining parties. This is related to the fact that all inclination vectors in $\widehat{I}$ count for a Failure of CHU, VVD and SGP, while for ARP, two of these inclination vectors are related to Success of ARP, and the remaining two are related to Failure of this party.

As one hopefully sees, the applications of the modified Hoede-Bakker index are very broad, and they may cover many situations. What is important here is to impose assumptions on the influence and probability distribution that will be appropriate for a given situation. Let us now illustrate in another example how the calculations on the modifications can be conducted. 
Table 4: Group decision for Figure 1

\begin{tabular}{c|c|c||c|c|c} 
inclination $i$ & $B i$ & $g d(B i)$ & inclination $i$ & $B i$ & $g d(B i)$ \\
\hline \hline$i^{N}=(1,1,1)$ & $(1,1,1)$ & +1 & $i^{\emptyset}=(-1,-1,-1)$ & $(-1,-1,-1)$ & -1 \\
\hline$i^{(1)}=(1,1,-1)$ & $(1,1,1)$ & +1 & $i^{(6)}=(-1,-1,1)$ & $(-1,-1,-1)$ & -1 \\
\hline$i^{(2)}=(1,-1,1)$ & $(1,-1,1)$ & +1 & $i^{(5)}=(-1,1,-1)$ & $(-1,1,-1)$ & -1 \\
\hline$i^{(3)}=(-1,1,1)$ & $(-1,1,1)$ & +1 & $i^{(4)}=(1,-1,-1)$ & $(1,-1,-1)$ & -1
\end{tabular}

\subsection{Theoretical example}

We consider a very simple example of a three-voter social network presented in Figure 1.

Figure 1: Three-voter social network

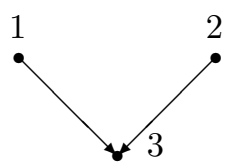

The set of voters is equal to $N=\{1,2,3\}$, and both voters 1 and 2 influence voter 3 . If voters 1 and 2 are unanimous with respect to their inclinations (that is, they have the same inclination), voter 3 will follow the influence of voters 1 and 2 . Otherwise, voter 3 will follow his own inclination. Moreover, suppose that the group decision is a simple majority. We can write then the decision vector:

$$
B i=b=\left(b_{1}, b_{2}, b_{3}\right)=\left\{\begin{array}{lll}
\left(i_{1}, i_{2}, i_{1}\right) & \text { if } & i_{1}=i_{2} \\
\left(i_{1}, i_{2}, i_{3}\right) & \text { if } & i_{1} \neq i_{2}
\end{array},\right.
$$

and the group decision

$$
\forall i \in I\left[g d(B i)=+1 \Leftrightarrow\left|\left\{k \in N \mid b_{k}=1\right\}\right| \geq 2\right] .
$$

Table 4 presents the group decision for our example.

Suppose now that we have a certain probability distribution $p$ over all eight inclination vectors. Using formula (16) of the modification $\Gamma_{k}$, we get the following:

$$
\begin{aligned}
& \Gamma_{1}(g d(B), p)=p\left(i^{N}\right)+p\left(i^{(1)}\right)+p\left(i^{(2)}\right)-p\left(i^{(3)}\right)-p\left(i^{(4)}\right)+p\left(i^{(5)}\right)+p\left(i^{(6)}\right)+p\left(i^{\emptyset}\right) \\
& \Gamma_{2}(g d(B), p)=p\left(i^{N}\right)+p\left(i^{(1)}\right)-p\left(i^{(2)}\right)+p\left(i^{(3)}\right)+p\left(i^{(4)}\right)-p\left(i^{(5)}\right)+p\left(i^{(6)}\right)+p\left(i^{\emptyset}\right) \\
& \Gamma_{3}(g d(B), p)=p\left(i^{N}\right)-p\left(i^{(1)}\right)+p\left(i^{(2)}\right)+p\left(i^{(3)}\right)+p\left(i^{(4)}\right)+p\left(i^{(5)}\right)-p\left(i^{(6)}\right)+p\left(i^{\emptyset}\right) .
\end{aligned}
$$

Next, we like to check when modification $\Gamma_{k}$ coincides with $\Phi_{k}$, that is, when $\Gamma_{k}$ measures Decisiveness of voter $k \in N$. By virtue of Proposition 4.1, we get the following conditions:

$$
\begin{aligned}
& \left\{\begin{array} { l } 
{ \Gamma _ { 1 } ( g d ( B ) , p ) = \Phi _ { 1 } ( W , p ) } \\
{ \Gamma _ { 2 } ( g d ( B ) , p ) = \Phi _ { 2 } ( W , p ) } \\
{ \Gamma _ { 3 } ( g d ( B ) , p ) = \Phi _ { 3 } ( W , p ) }
\end{array} \Leftrightarrow \left\{\begin{array}{l}
p\left(i^{N}\right)-p\left(i^{(3)}\right)=p\left(i^{(4)}\right)-p\left(i^{\emptyset}\right) \\
p\left(i^{N}\right)-p\left(i^{(2)}\right)=p\left(i^{(5)}\right)-p\left(i^{\emptyset}\right) \\
p\left(i^{N}\right)-p\left(i^{(1)}\right)=p\left(i^{(6)}\right)-p\left(i^{\emptyset}\right)
\end{array} \Leftrightarrow\right.\right. \\
& p\left(i^{N}\right)+p\left(i^{\emptyset}\right)=p\left(i^{(3)}\right)+p\left(i^{(4)}\right)=p\left(i^{(2)}\right)+p\left(i^{(5)}\right)=p\left(i^{(1)}\right)+p\left(i^{(6)}\right)=\frac{1}{4} .
\end{aligned}
$$


For each probability distribution $p$ which satisfies $(41)$, the modification $\Gamma_{k}$, coinciding with Decisiveness of player $k$, is equal to

$$
\Gamma_{k}(g d(B), p)=\Phi_{k}(W, p)=\frac{1}{2} \text { for each } k \in\{1,2,3\}
$$

One of the probability distributions satisfying (41) is $p^{*}$ defined by equation (9). We can also consider the modifications $\Psi_{k}$ defined in (20). We have

$$
\begin{aligned}
& \Psi_{1}(g d(B), p)=p\left(i^{(1)}\right)+p\left(i^{(5)}\right)+p\left(i^{(2)}\right)+p\left(i^{(6)}\right) \\
& \Psi_{2}(g d(B), p)=p\left(i^{(1)}\right)+p\left(i^{(4)}\right)+p\left(i^{(3)}\right)+p\left(i^{(6)}\right) \\
& \Psi_{3}(g d(B), p)=p\left(i^{(2)}\right)+p\left(i^{(4)}\right)+p\left(i^{(3)}\right)+p\left(i^{(5)}\right)
\end{aligned}
$$

For each probability distribution $p$ which satisfies (41) we have,

$$
\Psi_{k}(g d(B), p)=\frac{1}{2} \text { for each } k \in\{1,2,3\} .
$$

Next, we calculate when $\Psi_{k}(g d(B), p)$ coincides with the Shapley-Shubik index $S h_{k}(W)$. By virtue of (29) and $(30)$, we get $\Psi_{k}\left(g d(B), p^{S h}\right)=S h_{k}(W)$ for each $k=1,2,3$ iff

$$
\begin{gathered}
\frac{1}{6} \leq p^{S h}\left(i^{\emptyset}\right) \leq \frac{1}{3} \\
p^{S h}(i)=\left\{\begin{array}{ccc}
\frac{1}{3}-p^{S h}\left(i^{\emptyset}\right) & \text { for } & i \in\left\{i^{(4)}, i^{(5)}, i^{(6)}\right\} \\
p^{S h}\left(i^{\emptyset}\right)-\frac{1}{6} & \text { for } & i \in\left\{i^{(1)}, i^{(2)}, i^{(3)}\right\} . \\
\frac{1}{2}-p^{S h}\left(i^{\emptyset}\right) & \text { for } & i=i^{N}
\end{array}\right.
\end{gathered}
$$

If the probability distribution satisfies $(42)$, then

$$
\Psi_{k}\left(g d(B), p^{S h}\right)=S h_{k}(W)=\frac{1}{3} \text { for each } k=1,2,3 .
$$

Moreover, by virtue of (21), we get formulas for the modification $\Psi_{k}^{+}$:

$$
\begin{aligned}
& \Psi_{1}^{+}(g d(B), p)=\frac{p\left(i^{(1)}\right)+p\left(i^{(2)}\right)}{p\left(i^{N}\right)+p\left(i^{(1)}\right)+p\left(i^{(2)}\right)+p\left(i^{(4)}\right)} \\
& \Psi_{2}^{+}(g d(B), p)=\frac{p\left(i^{(1)}\right)+p\left(i^{(3)}\right)}{\left.p\left(i^{N}\right)+p\left(i^{(1)}\right)+p\left(i^{(3)}\right)+p\left(i^{(5)}\right)\right)} \\
& \Psi_{3}^{+}(g d(B), p)=\frac{p\left(i^{(2)}\right)+p\left(i^{(3)}\right)}{p\left(i^{N}\right)+p\left(i^{(2)}\right)+p\left(i^{(3)}\right)+p\left(i^{(6)}\right)}
\end{aligned}
$$

By virtue of Proposition 5.2, $\Psi_{k}^{+}\left(g d(B), p^{S h}\right)=S h_{k}(W)$ for $k=1,2,3$ iff

$$
\begin{gathered}
0 \leq p^{S h}\left(i^{\emptyset}\right)<1 \\
p^{S h}(i)=\left\{\begin{array}{ccc}
\frac{2}{11} \cdot\left(1-p^{S h}\left(i^{\emptyset}\right)\right) & \text { for } & i \in\left\{i^{(4)}, i^{(5)}, i^{(6)}, i^{N}\right\} \\
\frac{1}{11} \cdot\left(1-p^{S h}\left(i^{\emptyset}\right)\right) & \text { for } & i \in\left\{i^{(1)}, i^{(2)}, i^{(3)}\right\}
\end{array},\right.
\end{gathered}
$$

and if (43) is satisfied, then

$$
\Psi_{k}^{+}\left(g d(B), p^{S h}\right)=S h_{k}(W)=\frac{1}{3} \text { for each } k=1,2,3 .
$$

Finally, from definition (22), we have

$$
\Psi_{1}^{-}(g d(B), p)=\frac{p\left(i^{(5)}\right)+p\left(i^{(6)}\right)}{p\left(i^{(3)}\right)+p\left(i^{(5)}\right)+p\left(i^{(6)}\right)+p\left(i^{\emptyset}\right)}
$$




$$
\begin{aligned}
& \Psi_{2}^{-}(g d(B), p)=\frac{p\left(i^{(4)}\right)+p\left(i^{(6)}\right)}{p\left(i^{(2)}\right)+p\left(i^{(4)}\right)+p\left(i^{(6)}\right)+p\left(i^{\emptyset}\right)} \\
& \Psi_{3}^{-}(g d(B), p)=\frac{p\left(i^{(4)}\right)+p\left(i^{(5)}\right)}{p\left(i^{(1)}\right)+p\left(i^{(4)}\right)+p\left(i^{(5)}\right)+p\left(i^{\emptyset}\right)} .
\end{aligned}
$$

By virtue of Proposition 5.3, $\Psi_{k}^{-}\left(g d(B), p^{S h}\right)=S h_{k}(W)$ for $k=1,2,3$ iff

$$
\begin{aligned}
& 0 \leq p^{S h}\left(i^{N}\right)<1 \\
& p^{S h}(i)=\left\{\begin{array}{llc}
\frac{1}{11} \cdot\left(1-p^{S h}\left(i^{N}\right)\right) & \text { for } & i \in\left\{i^{(4)}, i^{(5)}, i^{(6)}\right\} \\
\frac{2}{11} \cdot\left(1-p^{S h}\left(i^{N}\right)\right) & \text { for } & i \in\left\{i^{\emptyset}, i^{(1)}, i^{(2)}, i^{(3)}\right\}
\end{array} .\right.
\end{aligned}
$$

If (44) is satisfied, then we also have

$$
\Psi_{k}^{-}\left(g d(B), p^{S h}\right)=S h_{k}(W)=\frac{1}{3} \text { for each } k=1,2,3 .
$$

Finally, we want to calculate when $\Psi_{k}(g d(B), p)$ coincides with the non-normalized Holler-Packel index. In our example

$$
I^{m w}=\left\{i^{(1)}, i^{(2)}, i^{(3)}\right\},
$$

and hence, by virtue of Proposition 6.1 , we get $\Psi_{k}\left(g d(B), p^{H P}\right)=H P_{k}(W)$ for $k=1,2,3$, where

$$
p^{H P}(i)=\left\{\begin{array}{ccc}
\frac{1}{3} & \text { if } & i \in\left\{i^{(1)}, i^{(2)}, i^{(3)}\right\} \\
0 & \text { if } & i \in I \backslash\left\{i^{(1)}, i^{(2)}, i^{(3)}\right\}
\end{array} .\right.
$$

Then, if (45) is satisfied, then we get

$$
\Psi_{k}\left(g d(B), p^{H P}\right)=H P_{k}(W)=\frac{2}{3} \text { for each } k=1,2,3 .
$$

In these two examples, where the first one is based on real political data and the second one analyzes a simple three-player network, the reader could hopefully see the advantages and innovative aspects of the modifications of the Hoede-Bakker index proposed in this paper. Let us summarize these advantages in the Conclusions drawn below.

\section{Conclusions}

In this paper, we consider measures of power for a social network in which groups of players have either to accept or to reject a proposal. Each voter has an inclination either to say 'yes' or 'no', but he may also be influenced by other voter(s), and consequently his final decision may be different from his (original) inclination. To the best of our knowledge, the (generalized) Hoede-Bakker index was analyzed so far only under the assumption of equally probable inclination vectors. The main aim of this paper is to generalize the (generalized) Hoede-Bakker index by considering arbitrary probability distributions over all inclination vectors. First, we define several modifications of the generalized decisional power index for an arbitrary probability distribution. Next, by choosing 'proper' probability distributions, we construct measures that coincide with the Shapley-Shubik index and with the Holler-Packel index. Finally, in order to illustrate the notions introduced, we consider two simple examples.

What are the advantages and innovative aspects of the (modified) Hoede-Bakker index, and how can one apply the insights provided in the paper? The main advantages of the index seem to be related to the general character of the index. This generality comes from two elements in the framework: broadly defined influence between agents, and an arbitrary probability distribution over all inclination vectors. First of all, by allowing for an arbitrary probability distribution, the index can be applied to many situations: both real-life practical examples and hypothetical theoretical networks. With some probability distributions, the modifications of 
the Hoede-Bakker index lead to the standard power indices, but now defined in the framework of a social network with influence between agents. In particular, the generalized Hoede-Bakker index measures 'net' Success, but with the uniform probability distribution it also coincides with Decisiveness. Consequently, it simplifies calculations when determining different measures in a social network, giving, in particular, an immediate answer to the question of Decisiveness. It is sufficient to calculate Success of a player, which immediately gives Failure, and therefore also 'net' Success and Decisiveness.

Another innovative aspect of the modified Hoede-Bakker indices is that depending on how the influence function is defined, the framework may cover many different situations. In particular, different motivations of players, i.e., both office seeking and policy seeking motivations, can be modeled within this framework.

Furthermore, as argued in the Introduction, the Hoede-Bakker index, originally defined in a framework which takes preferences of parties into account, may also be re-defined without considering preferences.

In order to apply the presented concepts, one should first define well the situation which is analyzed, and describe its main features. First, can we identify parties' inclinations with respect to making a particular decision? And if so, are the players influenced by the others? Do they (sometimes) decide differently from their inclinations? Moreover, are all possible influence configurations equally probable? And if not, can we describe the probability distribution over them? These are, in particular, the questions, which should be answered before applying the insights provided in the paper. And when clear and appropriate answers can be given, one can proceed with making the required calculations.

\section{Acknowledgment}

I like to thank Prof. Adrian Van Deemen for his suggestions concerning the Dutch example, and Prof. Harrie De Swart for his suggestions for improving the paper.

\section{References}

[1] Banzhaf J (1965) Weighted Voting Doesn’t Work: a Mathematical Analysis, Rutgers Law Review 19: 317-343

[2] Braham M and Holler MJ (2005) The Impossibility of a Preference-Based Power Index, Journal of Theoretical Politics 17: 137-157

[3] Coleman JS (1971) Control of Collectivities and the Power of a Collectivity to Act, in: Social Choice, edited by Lieberman, Gordon and Breach, London

[4] Coleman JS (1986) Individual Interests and Collective Action: Selected Essays, Cambridge University Press

[5] Einy E (1985) On Connected Coalitions in Dominated Simple Games, International Journal of Game Theory 14: 103-125

[6] Garrett G and Tsebelis G (1996) An Institutional Critique of Intergovernmentalism, International Organization 50: 269-299

[7] Garrett G and Tsebelis G (1999a) Why Resist the Temptation of Power Indices in the European Union?, Journal of Theoretical Politics 11: 291-308

[8] Garrett G and Tsebelis G (1999b) More Reasons to Resist the Temptation of Power Indices in the European Union, Journal of Theoretical Politics 11: 331-338

[9] Garrett G and Tsebelis G (2001) Even More Reasons to Resist the Temptation of Power Indices in the European Union, Journal of Theoretical Politics 13: 99-105 
[10] Hoede C and Bakker R (1982) A Theory of Decisional Power, Journal of Mathematical Sociology 8: $309-322$

[11] Holler MJ (1982) Forming Coalitions and Measuring Voting Power, Political Studies 30: 262-271

[12] Holler MJ and Packel EW (1983) Power, Luck and the Right Index, Journal of Economics 43: 21-29

[13] Hosli M (1997) Voting Strength in the European Parliament: The Influence of National and Partisan Actors, European Journal of Political Research 31: 351-366

[14] Hosli M (2002) Preferences and Power in the European Union, Homo Oeconomicus 19: 311-326

[15] König T and Bräuninger T (1998) The Inclusiveness of European Decision Rules, Journal of Theoretical Politics 10: 125-142

[16] Laruelle A and Valenciano F (2005) Assessing Success and Decisiveness in Voting Situations, Social Choice and Welfare 24: 171-197

[17] Morgan MJ (1976) The Modelling of Governmental Coalition Formation: A Policy-based Approach with Interval Measurement, Doctoral Dissertation, University of Michigan

[18] Napel S and Widgrén M (2001) Inferior Players in Simple Games, International Journal of Game Theory 30: $209-220$

[19] Napel S and Widgrén M (2002) The Power of a Spatially Inferior Player, Homo Oeconomicus 19: $327-343$

[20] Napel S and Widgrén M (2004) Power Measurement as Sensitivity Analysis - A Unified Approach, Journal of Theoretical Politics 16: 517-538

[21] Napel S and Widgrén M (2005) The Possibility of a Preference-Based Power Index, Journal of Theoretical Politics 17: 377-387

[22] Peleg B (1981) Coalition Formation in Simple Games with Dominant Players, International Journal of Game Theory 10: 11-33

[23] Rae D (1969) Decision Rules and Individual Values in Constitutional Choice, American Political Science Review 63: 40-56

[24] Rusinowska A (2008) On the not-Preference-based Hoede-Bakker Index, forthcoming in: Petrosjan and Mazalov (Eds.) Game Theory and Applications, Vol. XIII, Nova Science Publishers, Inc. New York

[25] Rusinowska A and De Swart H (2006) Generalizing and Modifying the Hoede-Bakker Index, In: De Swart et al. (Eds.) Theory and Applications of Relational Structures as Knowledge Instruments, No 2. Springer's Lecture Notes in Artificial Intelligence LNAI 4342, pp. 60-88, Springer, Heidelberg, Germany

[26] Rusinowska A and De Swart H (2007) On Some Properties of the Hoede-Bakker Index, Journal of Mathematical Sociology 31(4): 267-293

[27] Shapley LS and Shubik M (1954) A Method for Evaluating the Distribution of Power in a Committee System, American Political Science Review 48: 787-792

[28] Steunenberg B, Schmidtchen D and Koboldt C (1999) Strategic Power in the European Union: Evaluating the Distribution of Power in Policy Games, Journal of Theoretical Politics 11: 339-366

[29] Tsebelis G and Garrett G (1996) Agenda Setting Power Indices, and Decision Making in the European Union, International Review of Law and Economics 16: 345-361 
[30] Van Deemen A (1989) Dominant Players and Minimum Size Coalitions, European Journal of Political Research 17: 313-332

[31] Van Deemen A (1991) Coalition Formation in Centralized Policy Games, Journal of Theoretical Politics 3: $139-6161$

[32] Van Deemen A (1997) Coalition Formation and Social Choice, Boston: Kluwer Academic Publishers

[33] Van Roozendaal P (1993) Cabinets in the Netherlands: The Importance of 'Dominant' and 'Central' Parties, European Journal of Political Research 23: 35-54

\section{Appendix}

\section{Proof of Proposition 4.1}

By virtue of (11) and (14), we have $S=f(i)$ and $p(S)=p(i)$ for each $i \in I$. Moreover, $k \in S$ iff $i_{k}=+1$; $k \notin S$ iff $i_{k}=-1 ; S \in W$ iff $g d(B i)=+1, S \notin W$ iff $g d(B i)=-1$. Hence, by virtue of (1), we have then

$$
\Phi_{k}(W, p)=\sum_{\substack{i: i_{k}=+1 \\
g d(B i)=+1 \\
g d\left(B i^{k}\right)=-1}} p(i)+\sum_{\begin{array}{c}
i: i_{k}=-1 \\
g d(B i)=-1 \\
g d\left(B i^{k}\right)=+1
\end{array}} p(i)
$$

and therefore, since (6) is imposed,

$$
\Phi_{k}(W, p)=\sum_{\left\{i: i_{k}=+1\right\}} p(i) \cdot \frac{g d(B i)-g d\left(B i^{k}\right)}{2}+\sum_{\left\{i: i_{k}=-1\right\}} p(i) \cdot \frac{g d\left(B i^{k}\right)-g d(B i)}{2} .
$$

Hence,

$$
\begin{gathered}
\Gamma_{k}(g d(B), p)=\Phi_{k}(W, p) \Leftrightarrow \\
\sum_{\left\{i: i_{k}=+1\right\}} p(i) \cdot g d(B i)+\sum_{\left\{i: i_{k}=+1\right\}} p(i) \cdot g d\left(B i^{k}\right)-\sum_{\left\{i: i_{k}=-1\right\}} p(i) \cdot g d(B i)-\sum_{\left\{i: i_{k}=-1\right\}} p(i) \cdot g d\left(B i^{k}\right)=0 \Leftrightarrow \\
\sum_{\left\{i: i_{k}=+1\right\}} p(i) \cdot\left(g d(B i)+g d\left(B i^{k}\right)\right)-\sum_{\left\{i: i_{k}=+1\right\}} p\left(i^{k}\right) \cdot\left(g d\left(B i^{k}\right)+g d(B i)\right)=0 \Leftrightarrow \\
\sum_{\left\{i: i_{k}=+1\right\}}\left(p(i)-p\left(i^{k}\right)\right) \cdot\left(g d(B i)+g d\left(B i^{k}\right)\right)=0 .
\end{gathered}
$$

\section{Proof of Proposition 4.2}

By virtue of (20) and (46), we have

$$
\begin{gathered}
\Psi_{k}(g d(B), p)=\sum_{\left\{i: i_{k}=+1\right\}} \frac{p(i)+p\left(i^{k}\right)}{2} \cdot\left(g d(B i)-g d\left(B i^{k}\right)\right)= \\
=\sum_{\left\{i: i_{k}=+1\right\}} p(i) \cdot \frac{g d(B i)-g d\left(B i^{k}\right)}{2}+\sum_{\left\{i: i_{k}=+1\right\}} p\left(i^{k}\right) \cdot \frac{g d(B i)-g d\left(B i^{k}\right)}{2}= \\
=\sum_{\left\{i: i_{k}=+1\right\}} p(i) \cdot \frac{g d(B i)-g d\left(B i^{k}\right)}{2}+\sum_{\left\{i: i_{k}=-1\right\}} p(i) \cdot \frac{g d\left(B i^{k}\right)-g d(B i)}{2}=\Phi_{k}(W, p) .
\end{gathered}
$$

As stated in the proof of Proposition 4.1, $i_{k}=+1$ iff $k \in S$. Moreover, 
$\left(i_{k}=+1\right.$ and $g d(B i)=+1$ and $\left.g d\left(B i^{k}\right)=-1\right)$ iff $(k \in S \in W$ and $S \backslash\{k\} \notin W)$.

Hence, by virtue of (21) and (2),

$$
\Psi_{k}^{+}(g d(B), p)=\left(\sum_{\left\{i: i_{k}=+1\right\}} p(i) \cdot \frac{g d(B i)-g d\left(B i^{k}\right)}{2}\right) \cdot \frac{1}{\sum_{\left\{i: i_{k}=+1\right\}} p(i)}=\frac{\sum_{\substack{S: k \in S \in W \\ S \backslash\{k\} \notin W}} p(S)}{\sum_{S: k \in S} p(S)}=\Phi_{k}^{+}(W, p) .
$$

By analogy, by virtue of (22) and (3),

$$
\Psi_{k}^{-}(g d(B), p)=\left(\sum_{\left\{i: i_{k}=-1\right\}} p(i) \cdot \frac{g d\left(B i^{k}\right)-g d(B i)}{2}\right) \cdot \frac{1}{\sum_{\left\{i: i_{k}=-1\right\}} p(i)}=\frac{\sum_{\substack{S: k \notin S \notin W \\ S \cup\{k\} \in W}} p(S)}{\sum_{S: k \notin S} p(S)}=\Phi_{k}^{-}(W, p) .
$$

\section{Proof of Proposition 5.1}

By virtue of Laruelle and Valenciano (2005), $S h_{k}(W)=\Phi_{k}(W, p)$ for all $k$ and $W$ if

$$
p(S)=\frac{1}{n+1} \cdot \frac{1}{\left(\begin{array}{c}
n \\
|S|
\end{array}\right)} \text { for } S \subseteq N .
$$

Hence, applying (23) and (14), and replacing in (47) vote configuration $S$ by inclination vector $i$, and $|S|$ by $|i|$ (see (12)), we have for each $k \in N, \Psi_{k}\left(g d(B), p^{S h}\right)=\Phi_{k}\left(W, p^{S h}\right)=S h_{k}(W)$, where $p$ is defined in (31).

Moreover, by virtue of Laruelle and Valenciano (2005), if $p$ and $p^{\prime}$ are two probability distributions, then $\Phi_{k}(W, p)=\Phi_{k}\left(W, p^{\prime}\right)$ for every $k \in N$ and every voting rule $W$ iff for all $S \neq \emptyset$

$$
p^{\prime}(S)=p(S)+(-1)^{|S|+1} \cdot\left(p(\emptyset)-p^{\prime}(\emptyset)\right) .
$$

Hence, $\Psi_{k}(g d(B), p)=\Psi_{k}\left(g d(B), p^{\prime}\right)$ for every $k \in N$ and $g d(B)$ iff for each $i \neq i^{\emptyset}$

$$
p^{\prime}(i)=p(i)+(-1)^{|i|+1} \cdot\left(p\left(i^{\emptyset}\right)-p^{\prime}\left(i^{\emptyset}\right)\right) .
$$

By combining (31) and (49), we get (30). Taking into account that $0 \leq p(i) \leq 1$ for each $i \in I$, and applying this to (30), we get, for each $i \in I$ such that $|i|$ is even,

$$
0 \leq \frac{1}{n+1} \cdot\left(1-\frac{1}{\left(\begin{array}{c}
n \\
|i|
\end{array}\right)}\right) \leq p^{S h}\left(i^{\emptyset}\right) \leq 1-\frac{1}{n+1} \cdot\left(\frac{1}{\left(\begin{array}{c}
n \\
i \mid
\end{array}\right)}-1\right),
$$

and for each $i \in I$ such that $|i|$ is odd,

$$
\frac{1}{n+1} \cdot\left(\frac{1}{\left(\begin{array}{l}
n \\
|i|
\end{array}\right)}+1\right)-1 \leq p^{S h}\left(i^{\emptyset}\right) \leq \frac{1}{n+1} \cdot\left(\frac{1}{\left(\begin{array}{l}
n \\
|i|
\end{array}\right)}+1\right) \leq 1 .
$$

Note that for each $i \in I$,

$$
1-\frac{1}{n+1} \cdot\left(\frac{1}{\left(\begin{array}{c}
n \\
|i|
\end{array}\right)}-1\right) \geq 1, \quad \frac{1}{n+1} \cdot\left(\frac{1}{\left(\begin{array}{l}
n \\
|i|
\end{array}\right)}+1\right)-1 \leq 0 .
$$

Hence,

$$
\max _{|i|-\text { even }} \frac{1}{n+1} \cdot\left(1-\frac{1}{\left(\begin{array}{c}
n \\
|i|
\end{array}\right)}\right) \leq p^{S h}\left(i^{\emptyset}\right) \leq \min _{|i|-\text { odd }} \frac{1}{n+1} \cdot\left(\frac{1}{\left(\begin{array}{c}
n \\
|i|
\end{array}\right)}+1\right)
$$

and since

$$
\max _{|i|-\text { even }} \frac{1}{n+1} \cdot\left(1-\frac{1}{\left(\begin{array}{c}
n \\
|i|
\end{array}\right)}\right)=\frac{1}{n+1} \cdot\left(1-\frac{1}{\left(\begin{array}{c}
\operatorname{Even}\left(\left[\frac{n}{2}\right],\left[\frac{n}{2}\right]+1\right) \\
n+1
\end{array}\right)}\right),
$$




$$
\left.\min _{|i|-\text { odd }} \frac{1}{n+1} \cdot\left(\frac{1}{\left(\begin{array}{c}
n \\
|i|
\end{array}\right)}+1\right)=\frac{1}{n+1} \cdot\left(\frac{1}{\left(\operatorname{Odd}\left(\left[\frac{n}{2}\right],\left[\frac{n}{2}\right]+1\right)\right.}\right)+1\right),
$$

we get (29). Let us write (30) equivalently as

$$
p^{S h}(i)=\frac{1}{n+1} \cdot \frac{1}{\left(\begin{array}{c}
n \\
|i|
\end{array}\right)}+(-1)^{|i|+1} \cdot\left(\frac{1}{n+1}-p^{S h}\left(i^{\emptyset}\right)\right)
$$

for each $i \in I$. Note that, of course,

$$
\begin{aligned}
\sum_{i \in I} p^{S h}(i)=\sum_{i \in I} & {\left[\frac{1}{n+1} \cdot \frac{1}{\left(\begin{array}{c}
n \\
|i|
\end{array}\right)}+(-1)^{|i|+1} \cdot\left(\frac{1}{n+1}-p^{S h}\left(i^{\emptyset}\right)\right)\right]=\frac{1}{n+1} \cdot \sum_{i \in I} \frac{1}{\left(\begin{array}{c}
n \\
|i|
\end{array}\right)}+\left(\frac{1}{n+1}-p^{S h}\left(i^{\emptyset}\right)\right) \cdot \sum_{i \in I}(-1)^{|i|+1} } \\
& =\frac{1}{n+1} \cdot \sum_{|i|=0}^{|i|=n} \frac{1}{\left(\begin{array}{c}
n \\
|i|
\end{array}\right)} \cdot\left(\begin{array}{c}
n \\
|i|
\end{array}\right)+\left(\frac{1}{n+1}-p^{S h}\left(i^{\emptyset}\right)\right) \cdot \sum_{|i|=0}^{|i|=n}(-1)^{|i|+1} \cdot\left(\begin{array}{c}
n \\
|i|
\end{array}\right)=1 .
\end{aligned}
$$

\section{Proof of Proposition 5.2}

By virtue of Laruelle and Valenciano (2005), $S h_{k}(W)=\Phi_{k}^{+}(W, p)$ for all $k$ and $W$ if $p(\emptyset)=0$ and

$$
p(S)=\frac{\frac{1}{|S|}}{\sum_{t=1}^{n} \frac{1}{t}} \cdot \frac{1}{\left(\begin{array}{c}
n \\
|S|
\end{array}\right)} \text { for } S \neq \emptyset .
$$

Hence, applying (24) and (14), and replacing $S$ by $i$ in (50), we get, for each $k \in N, \Psi_{k}^{+}\left(g d(B), p^{S h}\right)=$ $\Phi_{k}^{+}\left(W, p^{S h}\right)=S h_{k}(W)$, where $p$ is defined in (33). Moreover, if $p$ and $p^{\prime}$ are two probability distributions, then (Laruelle and Valenciano 2005) $\Phi_{k}^{+}(W, p)=\Phi_{k}^{+}\left(W, p^{\prime}\right)$ for every $k \in N$ and every $W$ iff for all $S \neq \emptyset$

$$
\frac{p(S)}{1-p(\emptyset)}=\frac{p^{\prime}(S)}{1-p^{\prime}(\emptyset)}
$$

where $p(\emptyset), p^{\prime}(\emptyset)<1$. Hence, we get $(32)$. Note that, of course,

$$
\begin{gathered}
\sum_{i \in I} p^{S h}(i)=p^{S h}\left(i^{\emptyset}\right)+\sum_{i \in I \backslash\left\{i^{\emptyset}\right\}} p^{S h}(i)=p^{S h}\left(i^{\emptyset}\right)+\left(1-p^{S h}\left(i^{\emptyset}\right)\right) \cdot \sum_{i \in I \backslash\left\{i^{\emptyset}\right\}} \frac{\frac{1}{|i|}}{\sum_{t=1}^{n} \frac{1}{t}} \cdot \frac{1}{\left(\begin{array}{c}
n \\
|i|
\end{array}\right)}= \\
=p^{S h}\left(i^{\emptyset}\right)+\frac{1-p^{S h}\left(i^{\emptyset}\right)}{\sum_{t=1}^{n} \frac{1}{t}} \cdot \sum_{|i|=1}^{|i|=n} \frac{1}{|i|} \cdot \frac{1}{\left(\begin{array}{c}
n \\
|i|
\end{array}\right)} \cdot\left(\begin{array}{c}
n \\
|i|
\end{array}\right)=1 .
\end{gathered}
$$

\section{Proof of Proposition 5.3}

By virtue of Laruelle and Valenciano (2005), $S h_{k}(W)=\Phi_{k}^{-}(W, p)$ for all $k$ and $W$ if $p(N)=0$ and

$$
p(S)=\frac{\frac{1}{n-|S|}}{\sum_{t=1}^{n} \frac{1}{t}} \cdot \frac{1}{\left(\begin{array}{c}
n \\
|S|
\end{array}\right)} \text { for } S \neq N
$$

Hence, applying (25) and (14), and replacing $S$ by $i$ in (52), we get, for each $k \in N, \Psi_{k}^{-}\left(g d(B), p^{S h}\right)=$ $\Phi_{k}^{-}\left(W, p^{S h}\right)=S h_{k}(W)$, where $p^{S h}$ is defined in (35). Moreover, if $p$ and $p^{\prime}$ are two probability distributions, then (Laruelle and Valenciano 2005) $\Phi_{k}^{-}(W, p)=\Phi_{k}^{-}\left(W, p^{\prime}\right)$ for every $k \in N$ and every $W$ iff for all $S \neq \emptyset$

$$
\frac{p(S \backslash\{k\})}{1-p(N)}=\frac{p^{\prime}(S \backslash\{k\})}{1-p^{\prime}(N)},
$$


where $p(N), p^{\prime}(N)<1$. Hence, we get (34). Moreover, note that,

$$
\begin{gathered}
\sum_{i \in I} p^{S h}(i)=p^{S h}\left(i^{N}\right)+\sum_{i \in I \backslash\left\{i^{N}\right\}} p^{S h}(i)=p^{S h}\left(i^{N}\right)+\left(1-p^{S h}\left(i^{N}\right)\right) \cdot \sum_{i \in I \backslash\left\{i^{N}\right\}} \frac{\frac{1}{n-|i|}}{\sum_{t=1}^{n} \frac{1}{t}} \cdot \frac{1}{\left(\begin{array}{c}
n \\
|i|
\end{array}\right)}= \\
=p^{S h}\left(i^{N}\right)+\frac{1-p^{S h}\left(i^{N}\right)}{\sum_{t=1}^{n} \frac{1}{t}} \cdot \sum_{|i|=0}^{|i|=n-1} \frac{1}{n-|i|} \cdot \frac{1}{\left(\begin{array}{c}
n \\
|i|
\end{array}\right)} \cdot\left(\begin{array}{c}
n \\
|i|
\end{array}\right)=1 .
\end{gathered}
$$

\section{Proof of Proposition 6.1}

We have: $S=f(i) \in M(W)$ iff $i \in I^{m w}$. From Laruelle and Valenciano (2005), we have that $H P_{k}(W)=$ $\Phi_{k}\left(W, p_{W}\right)$ for each $k \in N$ and $W$, where

$$
p_{W}(S)=\left\{\begin{array}{ccc}
\frac{1}{m(W)} & \text { if } & S \in M(W) \\
0 & \text { if } & S \notin M(W)
\end{array} .\right.
$$

From (23), for each $k \in N, \Psi_{k}\left(g d(B), p^{H P}\right)=\Phi_{k}\left(W, p_{W}\right)=H P_{k}(W)$, where $p^{H P}(i)$ is defined in (38). 